\title{
Statistical-based database fingerprint: chemical space dependent representation of compound databases
}

\author{
Norberto Sánchez-Cruz and José L. Medina-Franco*
}

\begin{abstract}
Background: Simplified representation of compound databases has several applications in cheminformatics. Herein, we introduce an alternative and general method to build single fingerprint representations of compound databases. The approach is inspired on the previously published modal fingerprints that are aimed to capture the most significant bits of a fingerprint representation for a compound data set. The novelty of the herein proposed statistical-based database fingerprint (SB-DFP) is that it is generated based on binomial proportions comparisons taking as reference the distribution of "1" bits on a large representative set of the chemical space.

Results: To illustrate the Method, SB-DFPs were constructed for 28 epigenetic target data sets retrieved from a recently published epigenomics database of interest in probe and drug discovery. For each target data set, the SBDFPs were built based on two representative fingerprints of different design using as reference a data set with more than 15 million compounds from ZINC. The application of SB-DFP was illustrated and compared to other methods through association relationships of the 28 epigenetic data sets and similarity searching. It was found that SB-DFPs captured overall, the common features between data sets and the distinct features of each set. In similarity searching SB-DFP equaled or outperformed other approaches for at least 20 out of the 28 sets.
\end{abstract}

Conclusions: SB-DFP is a general approach based on binomial proportion comparisons to represent a compound data set with a single fingerprint. SB-DFP can be developed, at least in principle, based on any fingerprint and reference data set. SB-DFP is a good alternative for exploration of relationships between targets through its associated compound data sets and performing similarity searching.

Keywords: Chemical space, Epi-informatics, Molecular fingerprints, Representation, Similarity searching

\section{Background}

Molecular fingerprints are bit strings representations of chemical structures in which each position indicates the presence (1) or absence ( 0 ) of chemical features as defined in the design of the fingerprint. There are several types of molecular fingerprints described elsewhere $[1,2]$. Such representations are broadly employed for the assessment

\footnotetext{
*Correspondence: norberto.sc90@gmail.com; medinaj|@unam.mx Department of Pharmacy, School of Chemistry, Universidad Nacional Autónoma de México, Avenida Universidad 3000, 04510 Mexico City, Mexico

This work is dedicated to Dr. Gerald M. Maggiora on the occasion of his 80th Birthday.
}

of chemical space coverage, molecular diversity and similarity searching [1-3]. With the constant increasing size of chemical databases, such studies have become more computationally demanding, leading to the need of generating simplified representations of compound databases to optimize storage and calculation speed. To this end, many of the approaches that have been proposed generate a single fingerprint trying to capture the common chemical features presents in all compounds in a database (or at least in most of them). The first strategy dates back to 1996, when Shemetulskis et al. [4] employed the Daylight Chemical Information Systems, Inc. molecular fingerprint to build the so-called modal fingerprint, which contains the common bits found in 
the molecular fingerprints in a given compound data set. In the modal fingerprint, the degree to which bits have to be in common in the data set in order to be set as " 1 " is determined by a user-defined threshold value, which ranges from 50 to $100 \%$, being $50 \%$ the best performing threshold in different studies. Since 1996 the algorithm has been extended to different molecular fingerprints and a number of studies have shown its application in similarity searching $[5,6]$ and for the quantification of intra- and inter-database diversity [7]. In parallel, several modifications to this concept have been developed, mostly aiming to enhance its performance on similarity searches at the expense of increasing the complexity to implement the approach. Such approaches include bit scaling [810], bit silencing [11] and the determination of the best feature combinations [12]. In different publications the term "modal fingerprint" has been used to refer to distinct approaches. To avoid confusions, herein we refer as "database fingerprint (DFP)" to the modal fingerprint constructed using $50 \%$ as the predefined threshold.

In this work, we present the statistical-based database fingerprint (SB-DFP) as a novel and general approach to generate a compound database fingerprint based on binomial proportion comparisons. In this paper, we illustrate the application of SB-DFP in comparing targetassociated compound data sets and performing similarity searching. As a case study, and to further advance the emerging field of epi-informatics [13], the SB-DFPs were applied to a recently published epigenomics database with potential therapeutic significance.

\section{Methods}

\section{Concept and construction of SB-DFP}

As commented on the Background, in a "classic" DFP representation, to set a bit " 1 " requires that such bit is present in at least $50 \%$ of all the molecules in the input data set. The basic idea of such threshold is to extract common bits in at least half of the input data set. However, the underlying hypothesis assumes that the probability of presence of a feature (bit) in a molecular representation is $50 \%$ for each of them, so all bits are compared against such probability.

SB-DFP is based on the basic hypothesis that the probability of presence of a feature (bit) in a molecular representation is not equal for each bit. Instead, it is determined by the availability of such feature in a reference set e.g. the "known chemical space" (or a reasonable approximation) and such availability has to be determined. Once the frequency occurrence of each bit in a molecular representation is determined for both, namely the reference set and the data set of study, the SB-DFP is constructed by comparing the frequency occurrence of each bit between both sets. Thus, a bit is set to " 1 " only if the frequency in the target data set is statistically higher than the reference. Figure 1 depicts a schematic comparison between a classic DFP (reminiscent of the modal fingerprint, vide supra) and the SB-DFP, respectively. In this scheme the database fingerprint is illustrated for a short hypothetical fingerprint representation with 20-bit positions.

It should be noted that the SB-DFP representation for a given data set requires three main features (Fig. 1b): (1) a reference set, (2) a molecular fingerprint representation and 3) a statistical method to do the binomial proportion comparisons. The chosen features for this work are described below, although SB-DFP can be developed with different fingerprints, reference sets, and statistical methods.

\section{Compound data sets}

As a case study we generated SB-DFPs for a recently published epigenomics database [14]. The set of targets used as a test case in this work were selected based on their relevance in probe and epigenetic drug discovery that have attracted the attention to perform virtual screening $[15,16]$. However, the SB-DFP is general and could be used for other targets. The epigenomics database used in this study contains compounds associations against 60 epigenetic targets. For our analysis, we selected the information for 28 targets for which there was at least 50 reported compounds with a potency of $10 \mu \mathrm{M}$ or better. Table 1 summarizes the targets considered in this work that included bromodomain-containing proteins (BRD2, BRD3 and BRD4), histone acetyltransferases (CREBBP and EP300), DNA methyltransferase (DNMT1), histone lysine methyltransferase (EHMT2), histone deacetylases (HDAC1-HDAC11), lysine acetyltransferase (KAT2B), lysine demethylases (KDM1A and KDM4C), histone methyl-lysine binding proteins (L3MBTL1 and L3MBTL3), mitogen-activated protein kinase (MAP3K7), O-GlcNAcase (MGEA5), nuclear receptor coactivators with histone acetyltransferase activity (NCOA1 and NCOA3), and protein arginine methyltransferase (PRMT1). Table 1 also includes the number of compounds in each set (350 compounds on average with a maximum of 2740 for HDAC1). Note that SB-DFP could be applied to other data sets with larger number of compounds and their performance in, for instance, virtual screening, would need to be assessed in a case-by-case basis. It might be anticipated that the performance could be target-dependent as it happens in other virtual screening approaches.

\section{Reference set}

In this study, the All Clean subset from the ZINC12 database [17], with $16,403,844$ unique compounds, was 


\section{a DFP}

${ }^{1.0}\left[\begin{array}{llllllllllllllllllll}1 & 1 & 1 & 1 & 0 & 0 & 0 & 0 & 1 & 1 & 0 & 1 & 0 & 1 & 1 & 0 & 0 & 1 & 0 & 1\end{array}\right]$

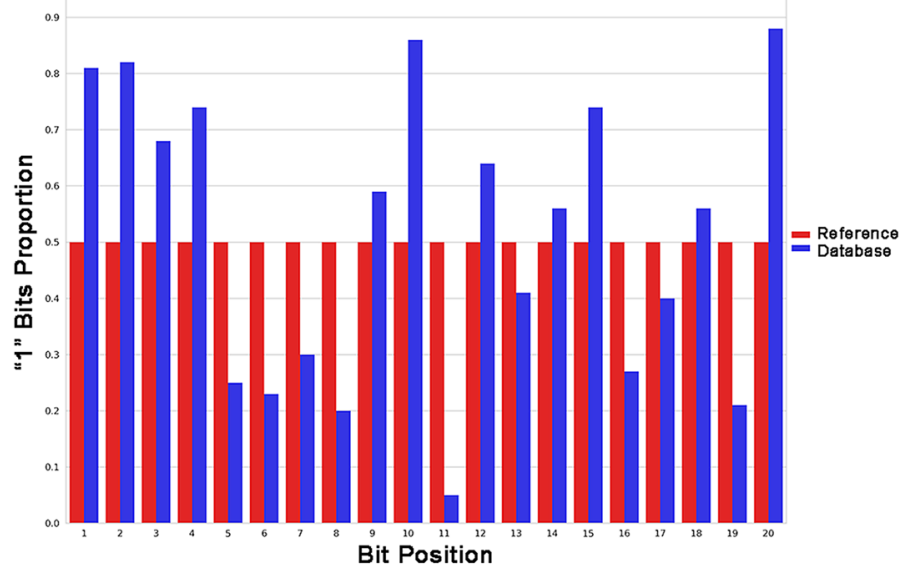

b SB-DFP

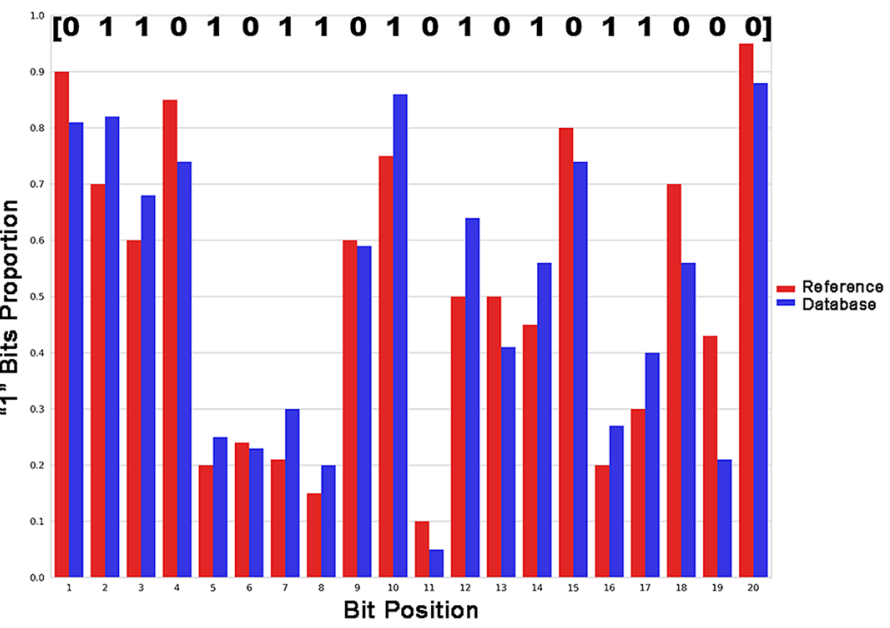

Fig. 1 Schematic representation of single fingerprints for a compound database and an hypothetical 20-bit fingerprint. The upper part of charts shows the binary representation of the generated single fingerprint: a database fingerprint (DFP) and $\mathbf{b}$ statistical-based database fingerprint (SB-DFP)

selected as starting point to build the reference set for SB-DFP calculations. We removed 21 compounds that could not be processed by the RDKit module for Python [18] and also 154 compounds present in the epigenomics database. The remaining molecules were randomly divided in two groups: one group with 1,000,000 compounds to be used as decoys in similarity searching (vide infra) and the second group with the remaining $15,403,690$ molecules to be used as reference for SB-DFP calculations. We employed such database with more than 15 million compounds as a representative sample of the currently known chemical space of small molecules available in ZINC. We emphasize that SB-DFP could be implemented using other reference data sets.

\section{Fingerprints}

We selected two fingerprints to illustrate the applicability of the concept of SB-DFP: Molecular ACCess System (MACCS) keys (166-bit) [19] as a "low resolution" dictionary fingerprint, and Extended Connectivity Fingerprint diameter 4 (ECFP4) as a "high resolution" representation [20] in its folded version of 2048 bits. MACCS keys and ECFP4 were generated with RDKit. 
Table 1 Selected datasets from the epigenomic database

\begin{tabular}{|c|c|c|c|c|c|c|c|c|c|}
\hline \multirow[t]{2}{*}{ Dataset } & \multirow[t]{2}{*}{$\begin{array}{l}\text { Number } \\
\text { of compounds }\end{array}$} & \multicolumn{2}{|c|}{$\begin{array}{l}\text { Intra-set similarity } \\
\text { median (Tc) }\end{array}$} & \multicolumn{2}{|c|}{ Average " 1 " bits } & \multicolumn{2}{|c|}{ Number of " 1 " bits in DFP } & \multicolumn{2}{|c|}{$\begin{array}{l}\text { Number of "1" bits } \\
\text { in SB-DFP }\end{array}$} \\
\hline & & MACCS $^{a}$ & ECFP4 $^{b}$ & MACCS $^{a}$ & ECFP $^{\text {b }}$ & MACCS $^{a}$ & ECFP4 $^{b}$ & MACCS $^{a}$ & ECFP4 $^{\mathrm{b}}$ \\
\hline BRD2 & 234 & 0.569 & 0.152 & 56.0 & 54.3 & 53 & 27 & 67 & 229 \\
\hline BRD3 & 246 & 0.573 & 0.153 & 56.6 & 54.6 & 53 & 26 & 73 & 231 \\
\hline BRD4 & 477 & 0.486 & 0.133 & 55.9 & 52.8 & 47 & 14 & 71 & 333 \\
\hline CREBBP & 105 & 0.694 & 0.276 & 56.1 & 53.9 & 52 & 36 & 50 & 185 \\
\hline DNMT1 & 127 & 0.403 & 0.115 & 55.4 & 51.7 & 50 & 13 & 62 & 281 \\
\hline EHMT2 & 61 & 0.636 & 0.228 & 62.4 & 55.7 & 62 & 41 & 56 & 167 \\
\hline EP300 & 57 & 0.425 & 0.106 & 58.2 & 55.7 & 53 & 11 & 56 & 285 \\
\hline HDAC10 & 190 & 0.514 & 0.165 & 53.2 & 50.6 & 50 & 17 & 46 & 272 \\
\hline HDAC11 & 137 & 0.494 & 0.156 & 51.2 & 50.8 & 48 & 16 & 42 & 229 \\
\hline HDAC1 & 2740 & 0.453 & 0.149 & 53.2 & 51.4 & 51 & 15 & 63 & 499 \\
\hline HDAC2 & 767 & 0.447 & 0.149 & 50.3 & 48.4 & 46 & 13 & 53 & 336 \\
\hline HDAC3 & 669 & 0.474 & 0.147 & 52.6 & 50.3 & 49 & 13 & 54 & 356 \\
\hline HDAC4 & 452 & 0.427 & 0.135 & 50.4 & 46.4 & 42 & 10 & 49 & 248 \\
\hline HDAC5 & 112 & 0.455 & 0.153 & 47.3 & 44.1 & 39 & 13 & 26 & 176 \\
\hline HDAC6 & 1374 & 0.474 & 0.149 & 54.3 & 49.8 & 48 & 13 & 62 & 415 \\
\hline HDAC7 & 112 & 0.489 & 0.165 & 50.4 & 45.8 & 43 & 12 & 28 & 197 \\
\hline HDAC8 & 864 & 0.500 & 0.153 & 54.9 & 51.2 & 50 & 12 & 52 & 398 \\
\hline HDAC9 & 102 & 0.494 & 0.169 & 52.6 & 47.4 & 46 & 13 & 29 & 190 \\
\hline KAT2B & 55 & 0.583 & 0.179 & 50.8 & 37.3 & 46 & 13 & 44 & 99 \\
\hline KDM1A & 241 & 0.380 & 0.143 & 44.8 & 46.2 & 31 & 21 & 31 & 216 \\
\hline KDM4C & 88 & 0.359 & 0.101 & 48.8 & 40.3 & 41 & 10 & 38 & 158 \\
\hline L3MBTL1 & 50 & 0.804 & 0.551 & 42.2 & 36.8 & 37 & 27 & 37 & 56 \\
\hline L3MBTL3 & 89 & 0.731 & 0.404 & 40.4 & 36.6 & 37 & 26 & 35 & 83 \\
\hline MAP3K7 & 96 & 0.539 & 0.137 & 57.1 & 60.5 & 59 & 35 & 45 & 190 \\
\hline MGEA5 & 67 & 0.683 & 0.316 & 54.2 & 39.6 & 48 & 19 & 42 & 126 \\
\hline NCOA1 & 51 & 0.350 & 0.105 & 45.5 & 43.3 & 34 & 11 & 18 & 132 \\
\hline NCOA3 & 157 & 0.368 & 0.109 & 47.7 & 44.6 & 39 & 10 & 26 & 166 \\
\hline PRMT1 & 61 & 0.395 & 0.076 & 53.0 & 53.5 & 41 & 9 & 40 & 239 \\
\hline Average & 350 & 0.507 & 0.178 & 52 & 48 & 46 & 18 & 46 & 232 \\
\hline
\end{tabular}

a MACCS keys 166-bit

b ECFP4 2048-bit

\section{Binomial proportion comparisons}

To perform the binomial proportion comparisons we employed a Z-test, as implemented in the statsmodels [21] module for Python. As can be found elsewhere [22], the proportion comparison relies on the calculation of a test statistic (called $Z_{\text {test }}$ ) defined as:

$$
Z_{\text {test }}=\frac{p_{t}-p_{r}}{\sqrt{\boldsymbol{P}(1-\boldsymbol{P})\left(\frac{1}{n_{t}}+\frac{1}{n_{r}}\right)}}
$$

where $p_{t}$ and $p_{r}$ are the proportions in which a given bit appears as " 1 " in the target and reference data sets for a total of $n_{t}$ and $n_{r}$ observations, respectively. $\boldsymbol{P}$ is the estimated true proportion of " 1 " bits considering both sample observations and it is calculated as:

$$
\boldsymbol{P}=\frac{n_{\boldsymbol{t}} p_{t}+n_{\boldsymbol{r}} p_{r}}{n_{t}+n_{r}}
$$

With the $Z_{\text {test }}$ calculated and through the standard Normal distribution, the exact probability than the observed difference between proportion is due to random variation can be determined (the $p$ value). So that the proportion difference is statistically significative if the $p$ value is lower than the associated to the confidence level selected a priori. For example, for the bit 100 in MACCS fingerprint, the bit " 1 " occurrence in the reference set is 
$10,892,579$ from $15,403,690$ observations $\left(p_{r}=0.707\right)$. By selecting a confidence level of $99 \%(p$ value $<0.01)$ and doing the calculations one gets that for a target data set of 350 compounds, the bit occurrence must be equal or greater than $268\left(p_{t}=0.766, p\right.$ value $\left.=0.008\right)$ to be set as an "1" bit in the SB-DFP representation even when for a bit occurrence of 248 the proportion seems to be larger $\left(p_{t}=0.708, p\right.$ value $\left.=0.476\right)$. This example illustrates that a greater proportion of " 1 " in a given bit for the target data set in comparison to the reference data set does not necessarily implies that such bit will be set as " 1 " in the SB-DFP. In other words, the proportion difference must be "big enough".

For this work we choose a confidence level of $99 \%(p$ value $<0.01$ ) based on the average AUC values obtained from similarity searching for ECFP4 and MACCS keys at five different confidence levels (vide infra). For the sets of targets and the fingerprints explored, the best performing method is the one with a confidence level of $99 \%$ (Additional file 1: Table S1) and all further calculations and discussion are based on such method. Of note, other $p$ values could be chosen for other targets and/or other fingerprints.

\section{SB-DFP to study inter-data set relationships}

To evaluate the performance of SB-DFP to capture the differences between data sets we calculated both, the classic DFP and the SB-DFP for each of the 28 targets. Both database fingerprints were constructed based on ECFP4 and MACCS keys fingerprints. Using the Tanimoto coefficient [23] and for each molecular fingerprint, we constructed the similarity matrices between epigenetic targets with three methodologies to calculate the similarity between pairs of targets: the median similarity between all-compound comparisons (ACC) in the data sets, the similarity between DFPs, and the similarity between SB-DFPs. This led to a total of six representations herein referred as ACC/MACCS, ACC/ECFP4, DFP/MACCS, DFP/ECFP4, SB-DFP/MACCS and SB$\mathrm{DFP} / \mathrm{ECFP} 4$. The range of similarity values for each representation was taken as a measure of its resolution. All six similarity matrices were transformed to their corresponding distance matrices based on the relationship (distance $=1-$ similarity). The distance matrices were used as basis for hierarchical clustering with complete linkage to analyze the ability of the representations to recover the known relationships between epigenetic targets based on its sequence identity. Such ability was assessed by calculating the Adjusted Rand Index (ARI) of each clustering [24] at a level of 10 clusters. The ARI measures the similarity between a given clustering and a ground truth: an ARI value of 1 indicates that the clustering recovers the original groups and an ARI value of 0 indicates random assignations. As ground truth, we used the hierarchical clustering with complete linkage obtained from the distance form of the sequence identity matrix (shown as Additional file 1: Table S10) as obtained from the alignment with Clustal Omega [25] with default parameters for the 28 targets studied. Sequences for all targets were taken from the Universal Protein Knowledgebase (UniProt) [26]. In addition, the number of "1" bits present in each representation was calculated as an approach of the amount of information contained in each one.

\section{SB-DFP as query for similarity searching}

Previous studies have shown that using single fingerprint representation of compound databases as query yield better results in similarity searching than fingerprint representations of single compounds [5, 6]. However, when single fingerprint representations are compared with methods that use information for multiple compound in a database, such as k-nearest neighbors ( $\mathrm{k}-\mathrm{NN})$ and binary kernel discrimination, the single fingerprint searches are outperformed [5]. In this work, we tested the performance of SB-DFP in similarity searching as compared to the classic DFP and 1-NN search strategies for both MACCS keys and ECFP4 fingerprints, methods such as binary kernel discrimination were not compared in this work given its reported lack of efficiency [5]. The Tanimoto coefficient was used as similarity measure, although other similarity metrics could be explored. For SB-DFP, five different confidence levels were tested for binomial proportion comparisons, here we report only the best performing one (99\%), the rest are summarized in Additional file 1: Table S1.

Using an approach similar to the one reported by Heikamp et al. [27], from each of the 28 epigenetic targets, 100 random sets of 10 active compounds each were randomly selected and used as query. In each case, all remaining active compounds were added as active database of compounds (ADCs) to a database containing one million compounds randomly selected from the ZINC All Clean subset (vide supra), called the search set. For the searches involving DFP and SB-DFP, the 10 compounds used as query were employed to build the corresponding single fingerprint, which was compared against all compounds in the search set, leading directly to a single similarity value per compound. On the other hand, for 1-NN, each of the compounds in the search set was compared to the 10 compounds used as query, leading to 10 similarity 
values per compound, from which the highest value was taken. For each similarity search, the compound recovery rates (RR) were calculated in a target-specific selection over the number of available ADCs as a measure of early enrichment. Receiver operating characteristic (ROC) curves and ROC area under the curve (AUC) values were also computed.

\section{Results and discussion}

\section{Bit proportions in the reference set}

As detailed in the Methods section, 15,403,690 compounds from the ZINC All Clean subset were taken as a representative sample of the currently known chemical space of small molecules. For the complete data set, the frequency of each bit was calculated for ECFP4 and MACCS keys. The results are summarized in Additional file 1: Tables S2 and S3. Of note, only 43 out of 166 bits for MACCS keys and 12 out of 2048 bits for ECFP4 have frequencies over 0.5 . This means that 43 and 12 bits of MACCS keys and ECFP4, respectively, are the most likely to appear in the DFP representation of any data set. Such bias is avoided in SB-DFP.

\section{Compound data sets}

For the 28 data sets studied in this work a total of six representations were generated for each set: the fingerprints for each compound, the single DFP, and SB-DFP, all based on ECFP4 and MACCS keys, respectively. Of note, the data sets representations based on DFP and SB-DFP have the advantage over "all-compounds" representation in that the speed of calculation is $N x M$ times faster than doing pairwise comparisons with all compounds in a set (with $N$ and $M$ being the number of compounds in two data sets).

The median of the intra-set similarity for all compounds in each data set was computed with MACCS keys and ECFP4 and the results are summarized in Table 1. Overall, all 28 sets have structural diverse compounds with, for instance, maximum median MACCS keys similarity of 0.694 (average of 0.507 ) and maximum median ECFP4 similarity of 0.551 (average of 0.178 ).

Table 1 also reports the average number of " 1 " bits for all compounds, as well as the number of " 1 " bits in the DFP and SB-DFP, respectively. For both MACCS keys and ECFP4 fingerprints, DFP representation has, on average, number of "1" bits (46 and 18, respectively) lower than all-compounds representation (52 and 48, respectively) but higher than the number of bits with occurrence frequencies over 0.5 in the reference set (vide supra). As expected, DFP contains less information than the complete data set. However, DFP captures more features in the data set than expected according to the occurrence frequencies in the reference data set.

DFP/MACCS and SB-DFP/MACCS capture similar amount of information with an average number of "1" bits of 46. However, as shown in Table 1, there is a dramatic increase in the number of "1" bits for SB-DFP/ ECFP4 as compared to DFP/ECFP4 (232 vs. 18). These results indicate that for the 28 data sets considered in this work, SB-DFP/ECFP4 captures a higher amount of specific structural features of the compounds.

\section{Similarity matrices}

The similarity matrices between epigenetic targets were calculated with three different approaches to calculate the similarity between pairs of targets: the median similarity of the all pairwise comparisons (e.g., all-compound comparisons) in the data sets (ACC), the similarity between their DFPs, and the similarity between their SB-DFPs, all based on MACCS keys and ECFP4 using the Tanimoto coefficient. As described in the Methods section, these representations are referred in this work as ACC/MACCS, ACC/ECFP4, DFP/MACCS, DFP/ECFP4, SB-DFP/MACCS, and SB-DFP/ECFP4. The six matrices are shown in Additional file 1: Tables S4-S9. Table 2 summarizes the maximum, minimum, average and range of Tanimoto similarity values for each similarity matrix. By using the median similarity between $\mathrm{ACC}$ in the data sets, the ranges are the smallest for MACCS keys and

Table 2 Range of Tanimoto similarity values in similarity matrices

\begin{tabular}{|c|c|c|c|c|c|c|c|c|}
\hline \multirow[t]{2}{*}{ Representation } & \multicolumn{4}{|c|}{ MACCS keys (166-bit) } & \multicolumn{4}{|c|}{ ECFP4 (2048-bit) } \\
\hline & Minimum & Average & Maximum & Range & Minimum & Average & Maximum & Range \\
\hline All compounds ${ }^{\mathrm{a}}$ & 0.293 & 0.407 & 0.804 & 0.511 & 0.059 & 0.114 & 0.553 & 0.494 \\
\hline DFP & 0.254 & 0.540 & 1.000 & 0.746 & 0.070 & 0.408 & 1.000 & 0.930 \\
\hline SB-DFP & 0.050 & 0.342 & 1.000 & 0.950 & 0.011 & 0.185 & 1.000 & 0.989 \\
\hline
\end{tabular}

a It should be noted that the comparisons involving the self-similarity of data sets does not reach a value of 1 and in some cases such self-similarity does not correspond to the highest value in the matrix row, that could be misinterpreted as the existence of pairs of databases more similar to each other than to themselves, which makes no sense. The matrices constructed by using DFP or SB-DFP do not present such problem, since when dealing with unique comparisons, a maximum of 1 is guaranteed for the diagonal of the matrix 
a

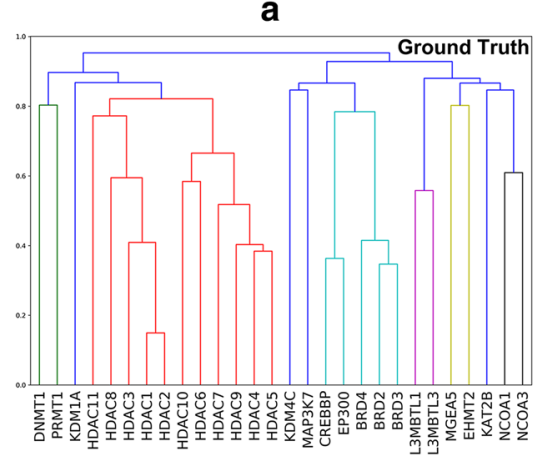

MACCS keys

b

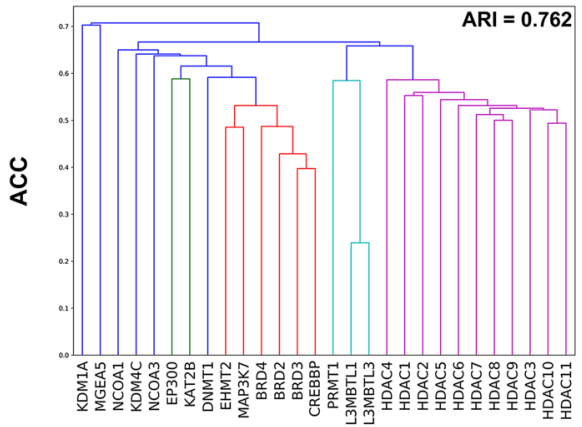

C

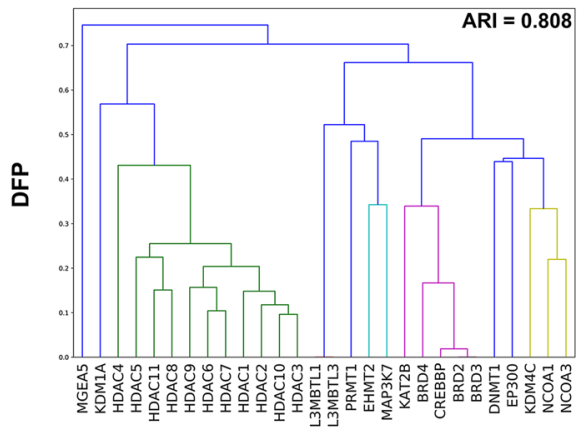

d

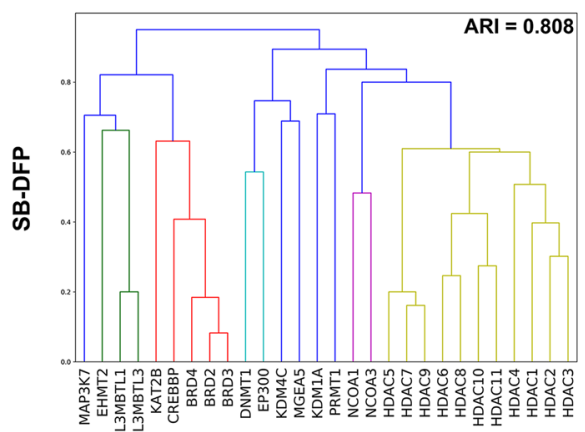

ECFP4

e

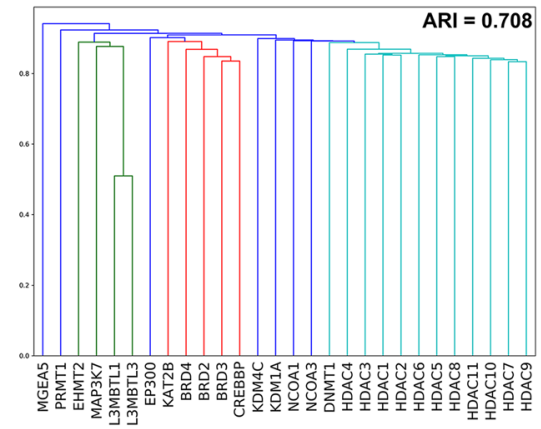

$\mathbf{f}$

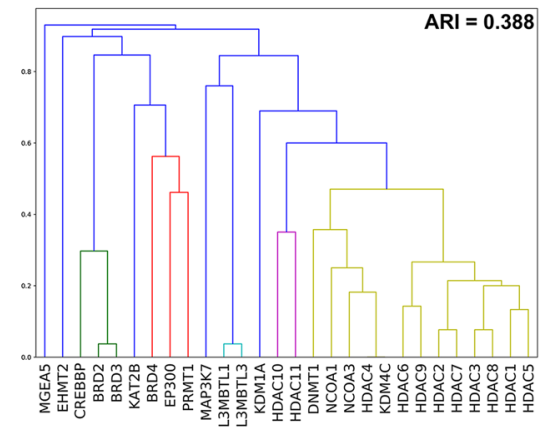

g

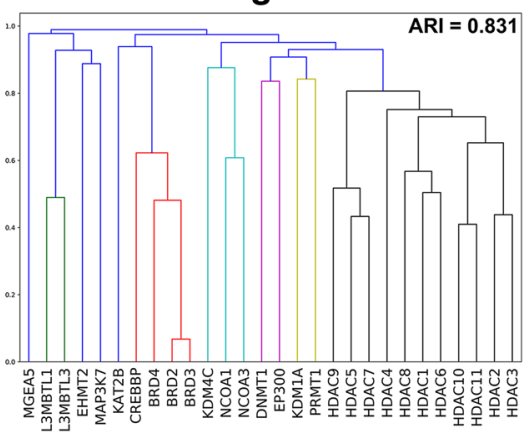

Fig. 2 Dendograms for hierarchical clustering of targets computed with different approaches based in two molecular fingerprints, MACCS keys and ECFP4. a The ground truth; $\mathbf{b}$, e all-compound comparisons (ACC); $\mathbf{c}$, $\mathbf{f}$ database fingerprint (DFP); $\mathbf{d}, \mathbf{g}$ statistical-based database fingerprint (SB-DFP). The Adjusted Rand Index (ARI) of each clustering is indicated in each panel. See main text for details 
a MACCs keys

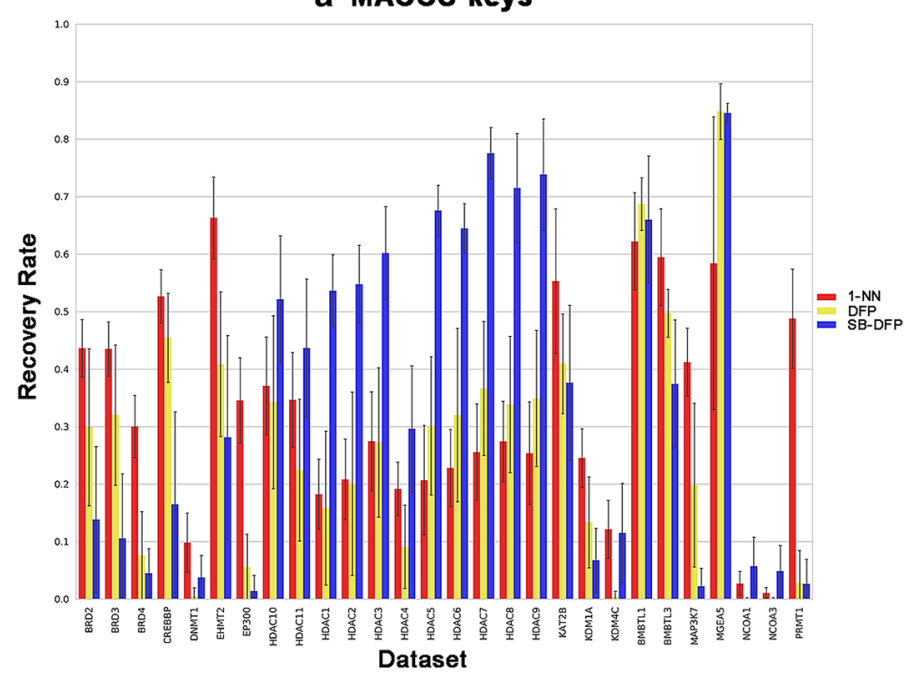

b ECFP4

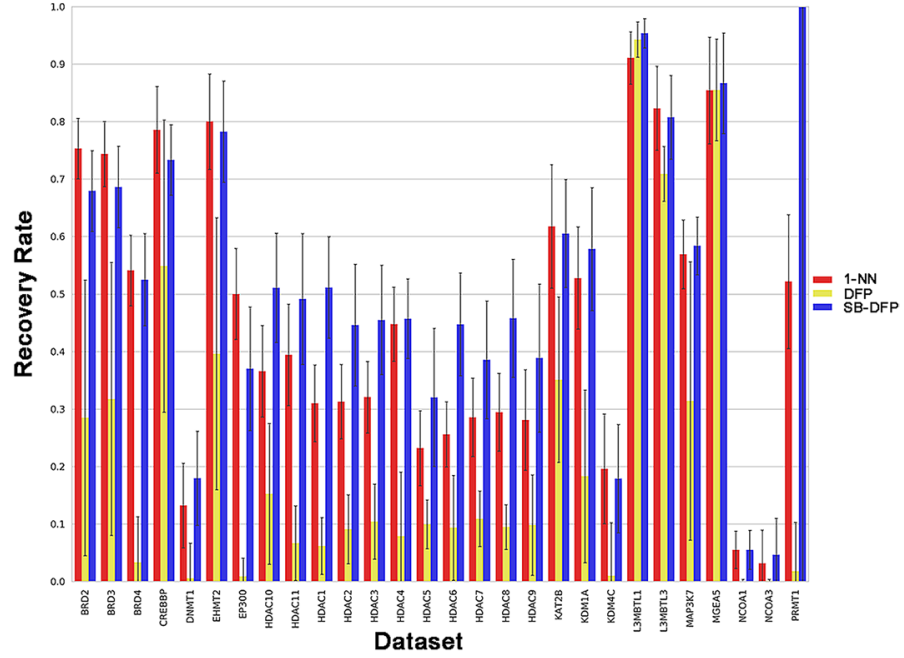

Fig. 3 Early enrichment performance of similarity searches. Average recovery rates (selection set size equal to the number of ADCs) for three search strategies over 28 epigenetic data sets are reported in a histogram representation for a MACCS keys and $\mathbf{b}$ ECFP4. Standard deviations are displayed as error bars

ECFP4 (i.e., 0.51 and 0.49 , respectively). Table 2 shows that the similarity matrices constructed using SB-DFP present a broader range of values $(0.950$ and 0.989$)$ than those constructed using DFP (0.746 and 0.930).

The SB-DFP matrices also have lower average similarities between data sets than the DFP matrices ( 0.540 vs. 0.342 for MACCS keys and 0.408 vs. 0.185 for ECFP4, respectively). Based on these results, the representation that captures better the differences between data sets is SB-DFP/ECFP4. This result agrees with the relative "higher resolution" of SB-DFP/ECFP4 i.e., higher number of " 1 " bits discussed above (Table 1).

\section{SB-DFP to study inter-data set relationship}

Figure 2 shows the dendrograms for each hierarchical clustering obtained with the corresponding distance matrices (vide supra). Analyzing the differences between data sets is not a trivial task and it is not straightforward evaluating the performance of a structural representation. In this work, we assessed the ability of the six representations listed above to recover the known relationships between epigenetic targets based on its sequence identity, using as metric the ARI at a level of 10 clusters and as ground truth the hierarchical clustering obtained from the distance form of the sequence 
a MACCs keys

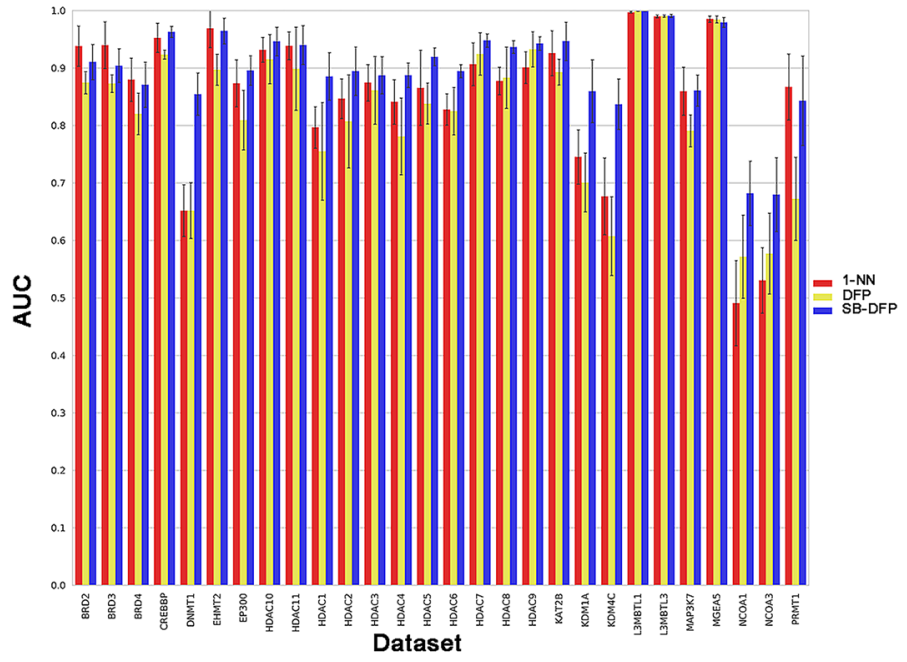

b ECFP4

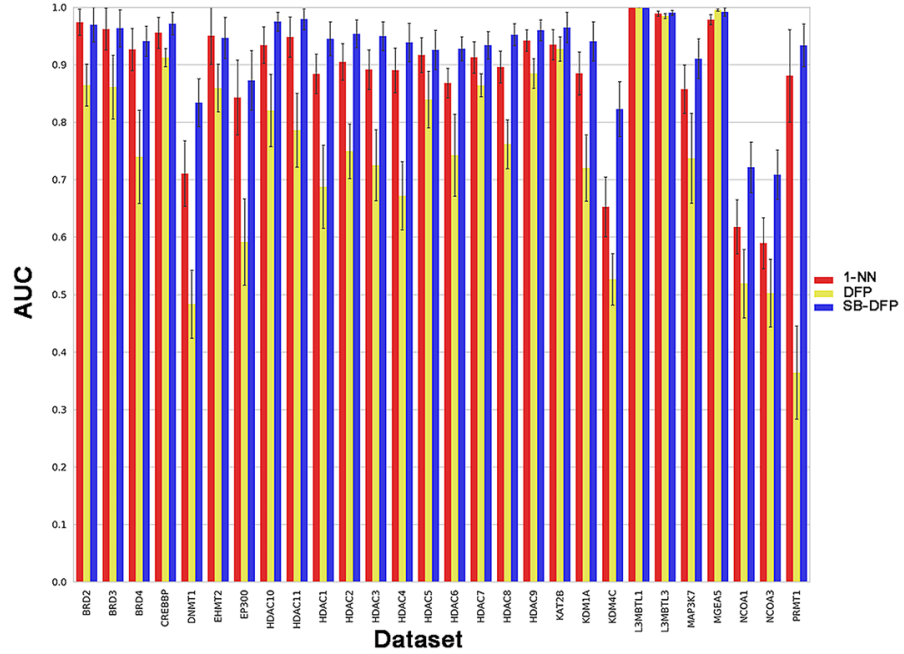

Fig. 4 General performance of similarity searches. Average AUCs for three search strategies over 28 epigenetic data sets are reported in a histogram representation for a MACCS keys and $\mathbf{b}$ ECFP4. Standard deviations are displayed as error bars

identity matrix (vide supra). The level of ten clusters was selected as ground truth given its recovery of four groups of epigenetic targets with known relationships: group 1 containing BRDs 2-4, CREBBP and EP300; group 2 containing HDACs 1-11; group 3 including L3MBTLs 1 and 3 ; and group 4 consisting of NCOAs 1 and 3. According to the results, the best performing methods were those based on the SB-DFP, with ARI values of 0.831 for SB-DP/ECFP4 and 0.808 for SB-DFP/MACCS. Methods based on ACC had worst but similar performances for both fingerprints with ARI values of 0.762 and 0.708 for ACC/MACCS and ACC/ECFP4 respectively. Finally, methods based on DFP had contrasting performances, being DFP/MACCS tied as the second best method with an ARI value of 0.808 and DFP/ECFP4 the worst of them with an ARI value of 0.388 .

\section{SB-DFP as template for similarity searching}

All 28 epigenetic data sets were subjected to systematic fingerprint search calculations. To obtain statistically relevant data, from each data set, 100 compound reference sets of 10 compounds were randomly selected and used as query in six different representations: the fingerprints for each compound (1-NN), the DFP and the SB-DFP, the three of them based on ECFP4 and MACCS keys. For the 
Table 3 Average recovery rates

\begin{tabular}{|c|c|c|c|c|c|c|}
\hline \multirow[t]{2}{*}{ Dataset } & \multicolumn{3}{|c|}{ MACCS keys (166-bit) } & \multicolumn{3}{|c|}{ ECFP4 (2048-bit) } \\
\hline & $1-\mathrm{NN}$ & DFP & SB-DFP & $1-\mathrm{NN}$ & DFP & SB-DFP \\
\hline BRD2 & $43.7(5.0)$ & $29.9(13.7)$ & $13.8(12.8)$ & $75.4(5.2)$ & $28.4(24.2)$ & $68.0(7.1)$ \\
\hline BRD3 & $43.5(4.8)$ & $32.0(12.3)$ & $10.6(11.3)$ & $74.4(5.7)$ & $31.9(23.8)$ & $68.7(7.1)$ \\
\hline BRD4 & $30.0(5.4)$ & $7.6(7.7)$ & $4.5(4.3)$ & $54.1(6.2)$ & $2.7(4.7)$ & $52.6(8.1)$ \\
\hline CREBBP & $52.7(4.7)$ & $45.5(7.8)$ & $16.5(16.2)$ & $79.0(5.4)$ & $55.6(25.0)$ & $73.7(4.2)$ \\
\hline DNMT1 & $9.9(5.2)$ & $0.5(1.5)$ & $3.8(3.9)$ & $12.9(5.7)$ & $0.0(0.0)$ & $17.7(7.1)$ \\
\hline EHMT2 & $66.3(7.1)$ & $40.9(12.6)$ & $28.1(17.8)$ & $80.1(8.0)$ & $40.2(23.5)$ & $78.4(8.3)$ \\
\hline EP300 & $34.6(7.5)$ & $5.5(5.8)$ & $1.4(2.7)$ & $50.2(7.7)$ & $0.7(2.8)$ & $37.0(10.8)$ \\
\hline HDAC10 & $37.1(8.6)$ & $34.2(15.1)$ & $52.2(11.1)$ & $36.5(8.0)$ & $15.4(12.3)$ & $51.1(9.5)$ \\
\hline HDAC11 & $34.7(8.3)$ & $22.5(12.4)$ & $43.7(12.1)$ & $39.6(8.8)$ & $6.6(6.4)$ & $49.3(11.3)$ \\
\hline HDAC1 & $18.2(6.1)$ & $15.8(13.5)$ & $53.7(6.3)$ & $30.9(6.7)$ & $6.3(5.1)$ & $51.1(9.0)$ \\
\hline HDAC2 & $20.9(7.0)$ & $20.1(16.1)$ & $54.8(6.9)$ & $31.3(6.5)$ & $9.1(6.0)$ & $44.7(10.6)$ \\
\hline HDAC3 & 27.5 (8.7) & $27.3(13.1)$ & $60.2(8.1)$ & $32.0(6.2)$ & $10.4(6.6)$ & $45.4(9.6)$ \\
\hline HDAC4 & $19.2(4.7)$ & $9.1(7.3)$ & $29.6(11.0)$ & $44.9(6.2)$ & $7.9(11.2)$ & $45.8(7.0)$ \\
\hline HDAC5 & 20.7 (9.6) & $30.2(12.1)$ & $67.6(4.4)$ & $23.1(6.4)$ & $10.0(4.3)$ & $32.0(12.1)$ \\
\hline HDAC6 & $22.8(6.7)$ & $32.0(15.1)$ & $64.5(4.3)$ & $25.7(5.8)$ & $9.3(9.1)$ & $44.6(9.0)$ \\
\hline HDAC7 & $25.6(8.4)$ & $36.6(11.7)$ & $77.6(4.5)$ & $28.4(6.7)$ & $11.0(4.9)$ & $38.6(10.4)$ \\
\hline HDAC8 & $27.4(7.0)$ & $33.9(11.9)$ & 71.5 (9.5) & 29.6 (6.9) & $9.5(3.9)$ & $46.2(9.8)$ \\
\hline HDAC9 & $25.4(9.0)$ & $34.9(11.9)$ & $73.9(9.7)$ & $27.7(7.5)$ & $9.6(8.7)$ & $38.4(13.0)$ \\
\hline KAT2B & $55.3(12.7)$ & $41.0(8.7)$ & $37.6(13.5)$ & $61.8(10.8)$ & $35.3(14.1)$ & $60.4(9.4)$ \\
\hline KDM1A & $24.6(5.1)$ & $13.3(8.0)$ & $6.8(5.6)$ & $53.3(8.4)$ & $18.3(15.1)$ & $58.4(10.0)$ \\
\hline KDM4C & $12.2(5.1)$ & $0.4(1.0)$ & $11.5(8.7)$ & $18.9(6.4)$ & $0.1(0.3)$ & $17.1(5.8)$ \\
\hline L3MBTL1 & $62.2(8.5)$ & $68.8(4.6)$ & $66.0(11.1)$ & $91.1(4.6)$ & 94.5 (1.8) & $95.5(2.3)$ \\
\hline L3MBTL3 & $59.5(8.5)$ & 49.7 (4.2) & $37.4(11.2)$ & $82.8(6.6)$ & $71.1(4.5)$ & $81.1(6.8)$ \\
\hline MAP3K7 & $41.2(6.0)$ & $19.8(14.3)$ & $2.2(3.1)$ & $56.6(5.2)$ & $31.1(23.8)$ & $58.0(4.0)$ \\
\hline MGEA5 & $58.5(25.6)$ & $84.8(4.9)$ & 84.6 (1.7) & $86.3(3.5)$ & $86.4(2.0)$ & $87.6(2.2)$ \\
\hline NCOA1 & $2.7(2.1)$ & $0.0(0.2)$ & $5.7(5.1)$ & $5.5(3.3)$ & $0.1(0.3)$ & $5.5(3.4)$ \\
\hline NCOA3 & $1.1(0.9)$ & $0.1(0.2)$ & $4.9(4.5)$ & $2.6(1.4)$ & $0.1(0.3)$ & $4.1(2.5)$ \\
\hline PRMT1 & 48.8 (8.7) & $2.8(5.7)$ & $2.7(4.3)$ & $52.8(10.5)$ & $1.0(3.8)$ & $55.3(12.1)$ \\
\hline Average & $33.1(19.6)$ & $26.4(22.8)$ & $35.3(29.1)$ & $46.0(25.9)$ & $21.5(28.4)$ & $50.2(23.8)$ \\
\hline
\end{tabular}

The best performing methods for each dataset are shown in bold. If there were no significative difference between two or more methods, all of them are marked. Standard deviations are shown in parentheses

six search strategies, Figs. 3 and 4 show the results of the RR and AUC, respectively. In terms of early enrichment, by using MACCS keys as molecular representation, the SB-DFP approach outperformed the other methods with an average RR of $35.3 \%$, followed by $1-\mathrm{NN}$ (33.1\%) and DFP (26.4\%). Similar trends were obtained using ECFP4, being the average RRs $50.2 \%, 46 \%$ and 21.5 for SB-DFP, $1-\mathrm{NN}$, and DFP respectively. Regarding to the global performance, the tendency was identical. The best performing method in both cases was SB-DFP, for MACCS keys with an average AUC of 0.898 , followed by $1-\mathrm{NN}$ and DFP with average AUCs of 0.853 and 0.824 respectively and for ECFP4 with average AUCs of 0.926, 0.882 and 0.755 for SB-DFP, $1-\mathrm{NN}$ and DFP respectively. These results revealed the anticipated differences between high- and low-resolution fingerprints, since ECFP4 achieved higher RRs and AUCs for 1-NN searches, while for the single fingerprint searches the higher values corresponded to the most populated representations in terms of number of bits " 1 " (MACCS keys for DFP and ECFP4 for SB-DFP).

The results also illustrated the general data set-dependence of the similarity searching performance and the good success rates achieved for 2D fingerprint methods, since the best performing search strategy for each data set obtained an average RR of at least $50 \%$ in 22 of 28 cases, and an average AUC larger than 0.7 in all of them. By analyzing the individual performances, according to RRs (Table 3), SB-DFP was the best method for 17 cases, from which eight were based on MACCS keys, seven 
Table 4 Average areas under ROC curves

\begin{tabular}{|c|c|c|c|c|c|c|}
\hline \multirow[t]{2}{*}{ Dataset } & \multicolumn{3}{|c|}{ MACCS keys (166-bit) } & \multicolumn{3}{|l|}{ ECFP4 } \\
\hline & $1-\mathrm{NN}$ & DFP & SB-DFP & $1-\mathrm{NN}$ & DFP & SB-DFP \\
\hline BRD2 & $0.938(0.035)$ & $0.875(0.019)$ & $0.911(0.031)$ & $0.974(0.023)$ & $0.865(0.037)$ & $0.970(0.030)$ \\
\hline BRD3 & $0.940(0.041)$ & $0.873(0.015)$ & $0.905(0.029)$ & $0.962(0.037)$ & $0.861(0.056)$ & $0.964(0.032)$ \\
\hline BRD4 & $0.880(0.038)$ & $0.821(0.036)$ & $0.871(0.040)$ & $0.927(0.037)$ & $0.740(0.082)$ & $0.941(0.026)$ \\
\hline CREBBP & $0.953(0.025)$ & $0.924(0.008)$ & $0.963(0.009)$ & $0.956(0.027)$ & $0.913(0.016)$ & $0.972(0.020)$ \\
\hline DNMT1 & $0.652(0.045)$ & $0.652(0.049)$ & $0.855(0.037)$ & $0.711(0.058)$ & $0.484(0.060)$ & $0.834(0.042)$ \\
\hline EHMT2 & $0.969(0.033)$ & $0.897(0.027)$ & $0.965(0.023)$ & $0.951(0.050)$ & $0.860(0.042)$ & $0.947(0.036)$ \\
\hline EP300 & $0.874(0.041)$ & $0.810(0.052)$ & $0.896(0.026)$ & $0.843(0.066)$ & $0.592(0.076)$ & $0.873(0.052)$ \\
\hline HDAC10 & $0.932(0.022)$ & $0.916(0.043)$ & $0.946(0.025)$ & $0.934(0.032)$ & $0.821(0.063)$ & $0.975(0.016)$ \\
\hline HDAC11 & $0.939(0.024)$ & $0.899(0.073)$ & $0.940(0.034)$ & $0.948(0.035)$ & $0.786(0.065)$ & $0.979(0.018)$ \\
\hline HDAC1 & $0.797(0.036)$ & $0.755(0.085)$ & $0.886(0.041)$ & $0.884(0.035)$ & $0.688(0.073)$ & $0.945(0.030)$ \\
\hline HDAC2 & $0.847(0.035)$ & $0.808(0.081)$ & $0.895(0.042)$ & $0.905(0.032)$ & $0.750(0.048)$ & $0.954(0.024)$ \\
\hline HDAC3 & $0.875(0.032)$ & $0.862(0.059)$ & $0.888(0.032)$ & $0.892(0.035)$ & $0.725(0.062)$ & $0.950(0.025)$ \\
\hline HDAC4 & $0.841(0.039)$ & $0.781(0.067)$ & $0.888(0.021)$ & $0.890(0.039)$ & $0.672(0.060)$ & $0.939(0.034)$ \\
\hline HDAC5 & $0.866(0.066)$ & $0.838(0.036)$ & $0.920(0.016)$ & $0.917(0.030)$ & $0.840(0.049)$ & $0.926(0.035)$ \\
\hline HDAC6 & $0.828(0.028)$ & $0.825(0.042)$ & 0.895 (0.011) & $0.868(0.026)$ & $0.743(0.072)$ & $0.928(0.021)$ \\
\hline HDAC7 & $0.907(0.037)$ & $0.925(0.037)$ & $0.948(0.012)$ & $0.913(0.027)$ & $0.864(0.020)$ & $0.934(0.024)$ \\
\hline HDAC8 & $0.878(0.024)$ & $0.883(0.054)$ & $0.937(0.011)$ & $0.896(0.028)$ & $0.762(0.043)$ & $0.953(0.019)$ \\
\hline HDAC9 & $0.901(0.028)$ & $0.933(0.031)$ & $0.943(0.012)$ & $0.942(0.019)$ & $0.885(0.026)$ & $0.960(0.018)$ \\
\hline KAT2B & $0.926(0.039)$ & $0.893(0.022)$ & $0.947(0.033)$ & $0.935(0.027)$ & $0.928(0.022)$ & $0.965(0.027)$ \\
\hline KDM1A & $0.745(0.048)$ & $0.701(0.051)$ & $0.860(0.055)$ & $0.885(0.038)$ & $0.721(0.058)$ & $0.941(0.034)$ \\
\hline KDM4C & $0.677(0.067)$ & $0.608(0.069)$ & $0.837(0.044)$ & $0.653(0.052)$ & $0.527(0.045)$ & $0.823(0.048)$ \\
\hline L3MBTL1 & $0.997(0.001)$ & $0.999(0.000)$ & $1.000(0.000)$ & $1.000(0.000)$ & $1.000(0.000)$ & $1.000(0.000)$ \\
\hline L3MBTL3 & $0.990(0.003)$ & $0.991(0.002)$ & $0.991(0.003)$ & $0.989(0.005)$ & $0.985(0.004)$ & $0.990(0.005)$ \\
\hline MAP3K7 & $0.860(0.042)$ & $0.791(0.028)$ & $0.861(0.027)$ & $0.858(0.042)$ & $0.738(0.079)$ & $0.911(0.035)$ \\
\hline MGEA5 & $0.985(0.005)$ & $0.985(0.006)$ & $0.979(0.009)$ & $0.979(0.009)$ & $0.996(0.002)$ & $0.992(0.007)$ \\
\hline NCOA1 & $0.491(0.074)$ & $0.572(0.073)$ & $0.682(0.056)$ & $0.618(0.047)$ & $0.519(0.060)$ & $0.722(0.044)$ \\
\hline NCOA3 & $0.530(0.057)$ & $0.577(0.071)$ & $0.680(0.064)$ & $0.590(0.045)$ & $0.503(0.059)$ & $0.709(0.043)$ \\
\hline PRMT1 & 0.867 (0.058) & $0.673(0.072)$ & $0.843(0.078)$ & $0.881(0.081)$ & $0.365(0.081)$ & $0.934(0.037)$ \\
\hline Average & $0.853(0.132)$ & $0.824(0.129)$ & $0.898(0.082)$ & $0.882(0.113)$ & $0.755(0.171)$ & $0.926(0.077)$ \\
\hline
\end{tabular}

The best performing methods for each dataset are shown in bold. If there were no significative difference between two or more methods, all of them are marked. Standard deviations are shown in parentheses

based on ECFP4 and two without significative difference between molecular fingerprints. The second best method was 1-NN with eight favorable cases by using ECFP4. For three data sets there was not significative difference between SB-DFP and 1-NN (Fig. 3). Additionally, the DFP representation was not the best performing method for any of the data sets studied.

According to the AUCs values (Table 4), the best performing method for 23 data sets was SB-DFP, from which four were based on MACCS keys, 17 based on ECFP4 and two without significative difference between fingerprints. The overall second-best approach was $1-\mathrm{NN}$ with better predictions for two data sets (one for each molecular fingerprint). In general, DFP had lower AUCs values as compared to the other two search methods (Table 4).
Remarkably, the search method based on SB-DFP could be applied in at least 20 out of the 28 data sets studied leading to the best RRs, with the additional advantage over 1-NN that the speed of calculation is $N$ times faster (with $N$ being the number of compounds used as query). This fact is because the number of comparisons needed for the screening is always equal to the number of compounds in the screened database in contrast to $1-\mathrm{NN}$, where this number scale with the number of compounds used as query.

\section{Conclusions and perspectives}

Here we presented the statistical-based database fingerprint (SB-DFP) as a novel general approach to generate single fingerprints of compound databases based 
on binomial proportion comparisons. In this work we shown its implementation for two molecular fingerprints (e.g., ECFP4 and MACCS keys) and one specific reference set (e.g., ZINC). However, the applicability of SB-DFP can be extended to any binary fingerprint and to other reference sets. Using as a case study a recently published set of 28 epigenetic compound sets with therapeutic relevance, we illustrate the application of SBDFP to capture the inter-data sets relationships and to perform similarity searching. For the data sets explored in this work the largest set has 2740 compounds (as deposited in ChEMBL) but SB-DFP could be applied to other larger compound data with relevance in drug or probe discovery. Despite the fact that no quantitative analysis was performed in terms of speed of calculation, it is clear that single fingerprint approaches to represent compound databases are faster because they depend on single rather than multiple comparisons.

Two major perspectives of the SB-DFP approach are application in high throughput virtual screening and target identification. To these ends, studies involving different molecular fingerprints, target-associated compound sets and reference data sets would be required, as well as exhaustive validations of their performance. Part of this work in ongoing and will be reported in due course.

\section{Additional file}

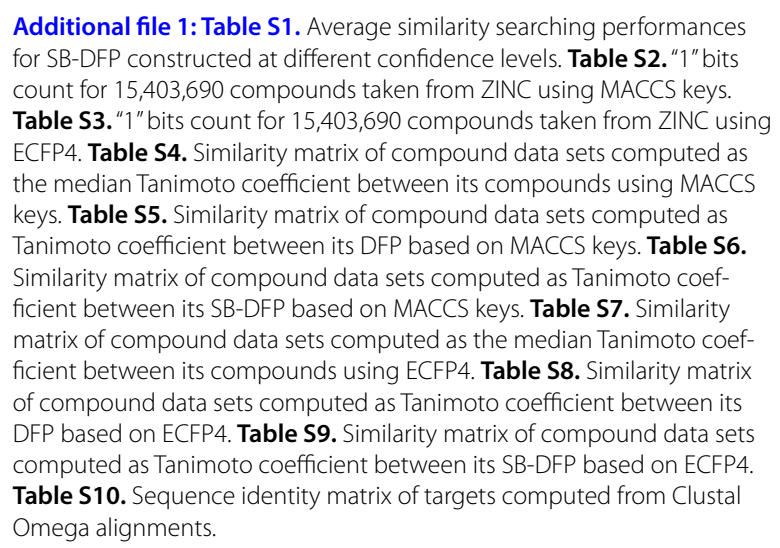
for SB-DFP constructed at different confidence levels. Table S2. "1" bits count for 15,403,690 compounds taken from ZINC using MACCS keys. Table S3. " 1 " bits count for 15,403,690 compounds taken from ZINC using ECFP4. Table S4. Similarity matrix of compound data sets computed as the median Tanimoto coefficient between its compounds using MACCS keys. Table S5. Similarity matrix of compound data sets computed as Tanimoto coefficient between its DFP based on MACCS keys. Table S6. Similarity matrix of compound data sets computed as Tanimoto coefficient between its SB-DFP based on MACCS keys. Table S7. Similarity matrix of compound data sets computed as the median Tanimoto coefficient between its compounds using ECFP4. Table S8. Similarity matrix of compound data sets computed as Tanimoto coefficient between its DFP based on ECFP4. Table S9. Similarity matrix of compound data sets computed as Tanimoto coefficient between its SB-DFP based on ECFP4. Table S10. Sequence identity matrix of targets computed from Clustal Omega alignments.

\section{Abbreviations}

ACC: all compound comparisons; ADC: active database of compounds; ARI: Adjusted Rand Index; AUC: area under the curve; DFP: database fingerprint; ECFP4: extended connectivity fingerprint of diameter four; $\mathrm{k}-\mathrm{NN}$ : $\mathrm{k}$-nearest neighbors; MACCS: molecular access system; ROC: receiver operating characteristic; RR: recovery rate; SB-DFP: statistical-based database fingerprint; UniProt: Universal Protein Knowledgebase.

\section{Authors' contributions}

All authors designed the study. NS-C performed the calculations. All authors wrote read and approved the final manuscript.

\section{Acknowledgements}

This work was funded by the Programa de Apoyo a Proyectos de Investigación e Innovación Tecnológica (PAPIIT) IA203718, Facultad de Química, UNAM. NS-C is thankful to CONACYT for the granted scholarship number 335997.

\section{Competing interests}

The authors declare that they have no competing interests.

\section{Availability of data and materials}

All datasets reported in the current study as well as the code implemented for calculations of DFP and SB-DFP are available in the GitHub repository: https:// github.com/DIFACQUIM/SB-DFP

\section{Funding}

This work was funded by the Programa de Apoyo a Proyectos de Investigación e Innovación Tecnológica (PAPIIT) IA203718.

\section{Publisher's Note}

Springer Nature remains neutral with regard to jurisdictional claims in published maps and institutional affiliations.

Received: 8 September 2018 Accepted: 14 November 2018

Published online: 22 November 2018

\section{References}

1. Cereto-Massagué A, Ojeda MJ, Valls C et al (2015) Molecular fingerprint similarity search in virtual screening. Methods 71:58-63. https://doi. org/10.1016/j.ymeth.2014.08.005

2. Muegge I, Mukherjee P (2016) An overview of molecular fingerprint similarity search in virtual screening. Expert Opin Drug Discov 11:137-148. https://doi.org/10.1517/17460441.2016.1117070

3. Heikamp K, Bajorath J (2012) Fingerprint design and engineering strategies: rationalizing and improving similarity search performance. Future Med Chem 4:1945-1959. https://doi.org/10.4155/fmc.12.126

4. Shemetulskis NE, Weininger D, Blankley CJ et al (1996) Stigmata: an algorithm to determine structural commonalities in diverse datasets. J Chem Inf Comput Sci 36:862-871. https://doi.org/10.1021/ci950169+

5. Hert J, Willett P, Wilton DJ et al (2004) Comparison of fingerprint-based methods for virtual screening using multiple bioactive reference structures. J Chem Inf Comput Sci 44:1177-1185. https://doi.org/10.1021/ ci034231b

6. Duan J, Dixon SL, Lowrie JF, Sherman W (2010) Analysis and comparison of 2D fingerprints: insights into database screening performance using eight fingerprint methods. J Mol Graph Model 29:157-170. https://doi. org/10.1016/j.jmgm.2010.05.008

7. Fernández-De Gortari E, García-Jacas CR, Martinez-Mayorga K, MedinaFranco JL (2017) Database fingerprint (DFP): an approach to represent molecular databases. J Cheminform 9:1-9. https://doi.org/10.1186/s1332 1-017-0195-1

8. Xue L, Stahura FL, Godden JW, Bajorath J (2001) Fingerprint scaling increases the probability of identifying molecules with similar activity in virtual screening calculations. J Chem Inf Comput Sci 41:746-753. https:// doi.org/10.1021/ci000311t

9. Xue L, Godden JW, Stahura FL, Bajorath J (2003) Profile scaling increases the similarity search performance of molecular fingerprints containing numerical descriptors and structural keys. J Chem Inf Comput Sci 43:1218-1225. https://doi.org/10.1021/ci030287u

10. Xue L, Stahura FL, Bajorath J (2004) Similarity search profiling reveals effects of fingerprint scaling in virtual screening. J Chem Inf Comput Sci 44:2032-2039. https://doi.org/10.1021/ci0400819

11. Wang Y, Bajorath $J$ (2008) Bit silencing in fingerprints enables the derivation of compound class-directed similarity metrics. J Chem Inf Model 48:1754-1759. https://doi.org/10.1021/ci8002045

12. Lounkine E, Hu Y, Batista J, Bajorath J (2009) Relevance of feature combinations for similarity searching using general or activity 
class-directed molecular fingerprints. J Chem Inf Model 49:561-570 https://doi.org/10.1021/ci800377n

13. Medina-Franco JL (2016) Epi-informatics: discovery and development of small molecule epigenetic drugs and probes. Academic Press, Cambridge. https://doi.org/10.1016/C2014-0-03789-6

14. Naveja JJ, Medina-Franco JL (2017) Insights from pharmacological similarity of epigenetic targets in epipolypharmacology. Drug Discov Today 23:141-150. https://doi.org/10.1016/j.drudis.2017.10.006

15. Lu W, Zhang R, Jiang $\mathrm{H}$ et al (2018) Computer-aided drug design in epigenetics. Front Chem 6:57. https://doi.org/10.3389/fchem.2018.00057

16. Prieto-Martinez FD, Medina-Franco JL (2018) Charting the Bromodomain BRD4: towards the identification of novel inhibitors with molecular similarity and receptor mapping. Lett Drug Des Discov 15:1002-1011. https://doi.org/10.2174/1570180814666171121145731

17. Irwin JJ, Sterling T, Mysinger MM et al (2012) ZINC: a free tool to discover chemistry for biology. J Chem Inf Model 52:1757-1768. https:// doi.org/10.1021/ci3001277

18. RDKit: open-source cheminformatics. http://www.rdkit.org. Accessed Nov 2018.

19. Durant JL, Leland BA, Henry DR, Nourse JG (2002) Reoptimization of MDL keys for use in drug discovery. J Chem Inf Comput Sci 42:12731280. https://doi.org/10.1021/ci010132r
20. Rogers D, Hahn M (2010) Extended-connectivity fingerprints. J Chem Inf Model 50:742-754. https://doi.org/10.1021/ci100050t

21. Seabold S, Perktold J (2010) Statsmodels: econometric and statistical modeling with python. In: Proceedings of 9th python in science conference, pp 57-61

22. LeBlanc D (2004) Statistics: concepts and applications for science. Jones \& Bartlett Publishers, Sudbury

23. Bajusz D, Rácz A, Héberger K (2015) Why is Tanimoto index an appropriate choice for fingerprint-based similarity calculations? J Cheminform 7:20. https://doi.org/10.1186/s13321-015-0069-3

24. Hubert L, Arabie P (1985) Comparing partitions. J Classif 2:193-218. https://doi.org/10.1007/BF01908075

25. Sievers F, Wilm A, Dineen D et al (2014) Fast, scalable generation of high-quality protein multiple sequence alignments using Clustal Omega. Mol Syst Biol 7:539. https://doi.org/10.1038/msb.2011.75

26. The UniProt Consortium (2017) UniProt: the universal protein knowledgebase. Nucl Acids Res 45:D158-D169. https://doi.org/10.1093/nar/gkw10 99

27. Heikamp K, Bajorath J (2011) Large-scale similarity search profiling of ChEMBL compound data sets. J Chem Inf Model 51:1831-1839. https:// doi.org/10.1021/ci200199u
Ready to submit your research? Choose BMC and benefit from:

- fast, convenient online submission

- thorough peer review by experienced researchers in your field

- rapid publication on acceptance

- support for research data, including large and complex data types

- gold Open Access which fosters wider collaboration and increased citations

- maximum visibility for your research: over $100 \mathrm{M}$ website views per year

At BMC, research is always in progress.

Learn more biomedcentral.com/submissions 